\title{
Sinks of Light Elements in Stars - Part I
}

\author{
Constantine P. Deliyannis \\ Indiana University, Astronomy Department, 319 Swain Hall West, 727 \\ E. 3rd Street, Bloomington, IN 47405-7105, USA \\ Marc H. Pinsonneault \\ The Ohio State University, Department of Astronomy, $140 \mathrm{~W}$. 18th \\ Ave. Columbus, Ohio 43210, USA \\ Corinne Charbonnel \\ Laboratoire d'Astrophysique de l'Observatoire Midi-Pyrénées, \\ CNRS-UMR 5572, 14, av.E.Belin, F-31400 Toulouse, France
}

\begin{abstract}
The fragile light elements lithium, beryllium, and boron $(\mathrm{Li}$, $\mathrm{Be}, \mathrm{B})$ are easily destroyed in stellar interiors, and are thus superb probes of physical processes occuring in the outer stellar layers. The light elements are also excellent tracers of the chemical evolution of the Galaxy, and can test big bang nucleosynthesis (BBN). These inter-related topics are reviewed with an emphasis on stellar physics.

In Part I (presented by CPD), an overview is given of the physical processes which can modify the surface abundances of the light elements, with emphasis on Population I dwarfs - convection; gravitational settling, thermal diffusion, and radiative levitation; slow mixing induced by gravity waves or rotation. We will discuss the increasingly large body of data which begin to enable us to discern the relative importance of these mechanisms in Population I main sequence stars. In Part II (presented by MHP), discussion is extended to the issue of whether or not the halo $\mathrm{Li}$ plateau is depleted, and includes the following topics: $\mathrm{Li}$ dispersion in field and globular cluster stars, Li production vs. destruction in Li-rich halo stars, and constraints from ${ }^{6} \mathrm{Li}$. Also discussed are trends with metal abundance and $T_{\text {eff }}$ and implications for chemical evolution and BBN. In Part III (presented by CC), evidence is reviewed that suggests that in situ mixing occurs in evolved low mass Population I and Population II stars. Theoretical mechanisms that can create such mixing are discussed, as well as their implications in stellar yields.
\end{abstract}

\section{Introduction}

In this three-part review we cover some of the most basic and important current topics of the subject; however, even in such an expanded review, it is not possible to do justice to all interesting topics. Part I complements Deliyannis (2000); 

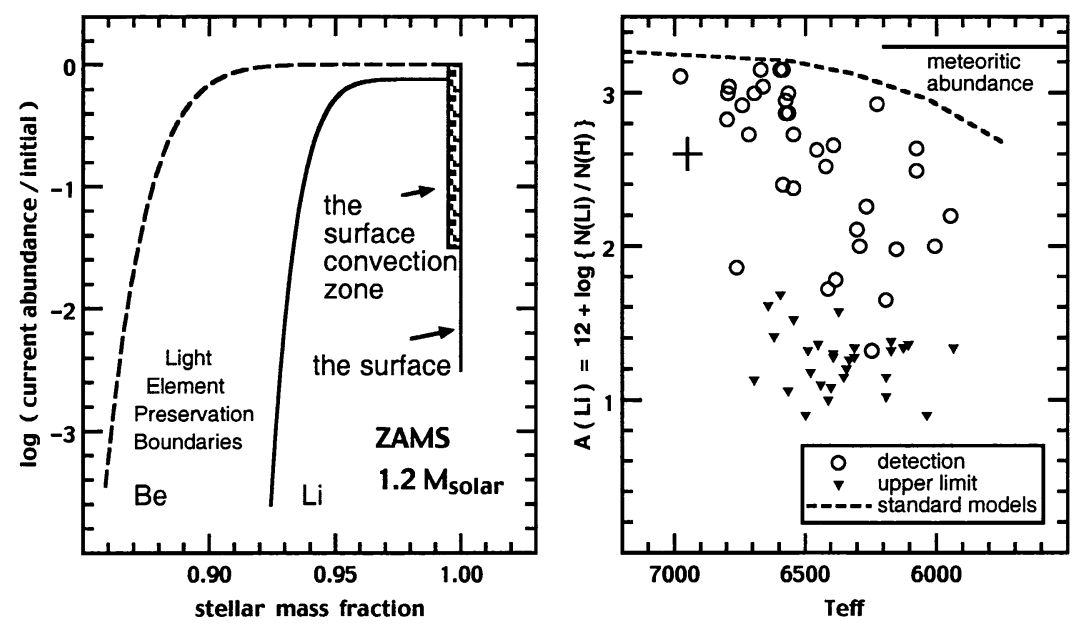

Figure 1. Left: The $\mathrm{Li}$ and Be preservation regions in a zams F-star. Right: Predictions of the classical theory compared to field star data. It is clear that additional physics is occuring inside these stars.

other recent reviews and points of view include Jeffries (2000), Pasquini (2000, these proceedings), Cayrel (1999), Martin (1997), and Pinsonneault (1997).

The light element tracers lithium, beryllium, and boron (hereafter $\mathrm{Li}, \mathrm{Be}$, and B) are excellent probes of physical processes occuring in stellar interiors, the chemical evolution of our Galaxy, and BBN. Within standard BBN, knowledge of the primordial abundances of these light elements (and also deuterium and the two stable helium isotopes) would constrain the Universal baryonic density (see Part II), the nature of dark matter, and ultimately, perhaps even some of the fundamental laws of nature. Delineation of the light element evolution with metal abundance can teach us about processes related to the general chemical evolution of the Galaxy, such as halo mass outflow and disk mass inflow, history of the cosmic ray spectrum, and the initial mass function. In stars, it is mainly the fragility of the light elements that makes them excellent tracers of physical processes occuring in stellar interiors. In Part I we focus on what $\mathrm{Li}, \mathrm{Be}$, and $\mathrm{B}$ tell us about Population I dwarfs and stars near the turnoff.

\section{The Observed Lithium Morphology}

The classical theory of stellar evolution, which assumes spherical symmetry and ignores possible effects due to rotation, diffusion, mass loss, magnetic fields, and so on, fails to explain nearly all observed Population $\mathrm{I} \mathrm{Li}, \mathrm{Be}$, and $B$ abundance patterns. Perhaps the most striking example is the Li gap in F stars, as illustrated in Figure 1. Inside stars, $\mathrm{Li}, \mathrm{Be}$, and $\mathrm{B}$ are destroyed by energetic protons 


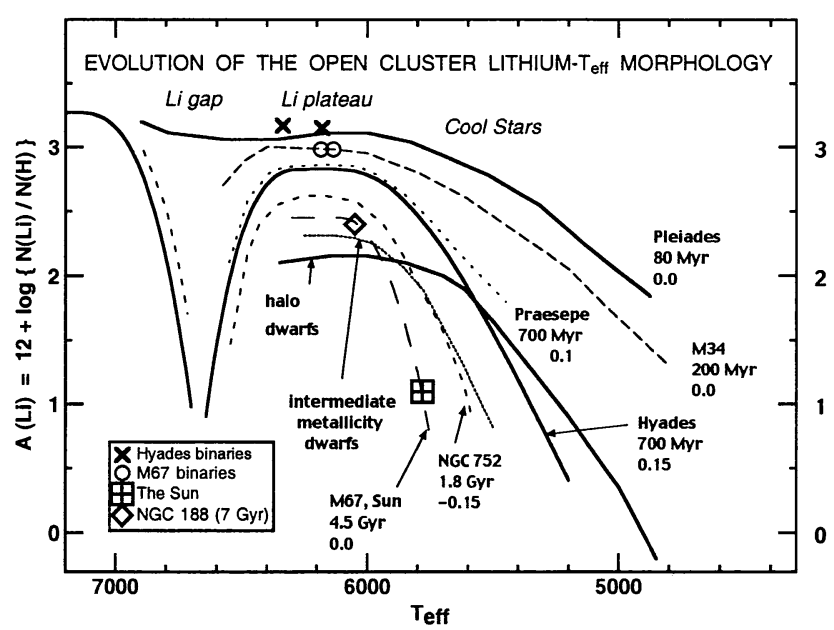

Figure 2. The main sequence Lithium Morphology of open clusters of different ages and composition, as indicated, of intermediate metallicity field stars, and of halo field stars.

at temperatures of only a few million $\mathrm{K}$, and thus survive only in the outermost layers. The left panel of Figure 1 shows the ${ }^{7} \mathrm{Li}$ and ${ }^{9} \mathrm{Be}$ profiles in a classical model of an F star that has arrived on the ZAMS (adapted from Deliyannis \& Pinsonneault 1997). ${ }^{9} \mathrm{Be}$ is sturdier than ${ }^{7} \mathrm{Li}$, and thus survives to about twice the depth; the B isotopes are even sturdier and survive to a bit over twice the Be-survival depth; and ${ }^{6} \mathrm{Li}$ is the most fragile of these isotopes and survives to about only half the ${ }^{7} \mathrm{Li}$ - survival depth. The surface convection zone (hereafter, SCZ) is much shallower than any of the light element preservation regions, and so it is impossible to deplete the surface light element abundances during the main sequence (see $\S 3$; the small amount of ${ }^{7} \mathrm{Li}$ depletion shown was a result of pre-main sequence destruction). Yet, in stark contrast to the strictures of the classical theory, the vast majority of field F and early G stars deplete their surface Li abundances, often quite severely (right panel of Figure 1; data from Boesgaard \& Tripicco 1986b). Clearly, interesting physics occurs in stars that is not included in the classical model.

An increasingly large body of data can help ascertain what the nature of this physics might be. Figure 2 (adapted from Deliyannis et al. 1998b) shows the Lithium Morphology (the mean trend of Li vs. $\mathrm{T}_{\text {eff }}$ ) in open clusters of different ages, in intermediate metallicity field stars, and in halo field stars. This figure encapsulates a tremendous amount of work carried out over the past 15 years. A partial list includes the following. Pleiades: Pilachowski et al. (1987), Boesgaard et al. (1988), and Soderblom et al. (1993a); M34: Jones et al. (1997); Hyades: Thorburn et al. (1993), and Boesgaard \& Tripicco (1986a); Praesepe: Soderblom et al. (1993b); NGC 752 and M67: preliminary analysis of the lead author's WIYN/Hydra data, for M67 see also Pasquini et al. (1997); intermediate metallicity and halo: see Ryan \& Deliyannis (1995). A more complete list of Li cluster work can be found in the excellent review by Jeffries (2000). The 
WIYN Open Cluster Study (Mathieu 2000) and several other groups are busily working at increasing the amount of information contained in this figure. It is clear that there are advantages to working with cluster data over working with field star data alone: whereas field stars have different compositions, ages, and evolutionary states, and probably as a result exhibit rather chaotic abundances (Figure 1), by contrast, a sample of cluster stars have the same (knowable) composition and age, and evolutionary state can be determined precisely. Thus, one can hope to find a well-defined pattern in cluster stars, as is indeed illustrated by Figure 2. Furthermore, one can study the evolution of the Lithium Morphology by studying clusters of different ages, and the dependence of the Lithium Morphology on composition. It should be noted that only the main trends for each cluster are shown here; in addition, there is evidence that spreads of $\mathrm{Li}$ exist at a given $\mathrm{T}_{\text {eff }}$. It has been argued that part of the spread (in the Pleiades, particularly) may be due to atmospheric effects, as opposed to real abundance differences (King et al. 1999), but evidence remains that at least some of the spread is intrinsic.

On the basis of the empirical content of Figure 2, Deliyannis (2000) proposed that mass (more or less $\mathrm{T}_{\text {eff }}$ ) is the first parameter that governs $\mathrm{Li}$ depletion in solar-type stars. Essentially, this reflects the striking Lithium Morphology exhibited in any given cluster. It was proposed that the second parameter is age, reflecting the apparent evolution of the Lithium Morphology, particularly main sequence Li depletion. Third parameters might include metallicity, initial rotation rates, etc. Theory (below) predicts metallicity-dependence of the $\mathrm{Li}$ depletion, but observations have not yet clearly established such a connection. However, some additional parameter may be required to explain the observed spreads of $\mathrm{Li}$ at a given $\mathrm{T}_{\text {eff }}$. Three main regions are evident in Figure 2: the Li Gap, the Li Plateau, and the Cool Stars. The challenge for theory is to explain these various empirical Li trends for dwarfs, as well as Li turnoff-subgiant observations ( $(5$; see also Part III), and Be and B data ( $(6)$. We consider each of these regions in more detail in $\S 4$; but first, we introduce various classes of light element depletion mechanisms in $§ 3$.

\section{Classical Models and Possible Missing Physics}

We argue that classical models fail in that they do not include certain physics that are clearly operating in real stars. The challenge is to identify, understand and properly describe this physics. Nonetheless, the classical theory provides the basic background. We thus review the fundamental aspects of $\mathrm{Li}$ depletion in classical models (see Deliyannis et al. 1990 for a detailed description of halo star models; low mass disk star models behave in a very similar way; see also Proffitt \& Michaud 1989, Pinsonneault et al. 1990, Swenson \& Faulkner 1992, Forestini 1994, D'Antona \& Mazzitelli 1994). In an evolutionary sequence of a one-solar mass model, it is necessary to begin evolution at the fully convective deuterium birthline. As models contract on the Hayashi track, the core becomes hot enough to destroy light elements (first ${ }^{6} \mathrm{Li}$, then ${ }^{7} \mathrm{Li}$, and then perhaps a tiny amount of ${ }^{9} \mathrm{Be}$ ). Convective mixing depletes the surface abundance. (To generalize, $A N Y$ physical mechanism that affects light element abundances at the base of the SCZ is nearly instantly evident at the surface as well. For example, diffusion 
out of or into the base of the SCZ decreases or increases, respectively, the surface abundance.) At about the same time, a radiative core develops, but light element destruction continues at the base of the SCZ (and thus surface) until the models reach the radiative Henyey track. The SCZ continues to get shallower (and cooler) essentially throughout the rest of the pre-main sequence (pre-MS) and main sequence, and there is no more surface $\mathrm{Li}$ depletion throughout this time. After the main sequence turnoff, the SCZ deepens and eventually sweeps past the boundary of Li preservation that was established during the main sequence. At this point, the fixed amount of $\mathrm{Li}$ mixes with material that has no $\mathrm{Li}$, and so the surface abundance is diluted. Note that the temperature at the base of the (now much deeper) SCZ is not high enough to destroy $\mathrm{Li}$, so dilution is the only Li depletion mechanism (see Part III). These are the only two ways to deplete the surface $\mathrm{Li}$ abundance in a classical sequence of $\mathrm{F}$ and $\mathrm{G}$ star models from the deuterium birthline up to the RGB tip: pre-MS destruction, and subgiant (and giant) dilution. There is a strong mass dependence: a pre-MS model of a later spectral type has a deeper convection zone that is hotter at its base, and will thus destroy more $\mathrm{Li}$ and for a longer period of time. This is the reason for the shape of the Li isochrone in Figure 1. For sufficiently cooler spectral types, main sequence $\mathrm{Li}$ depletion is also a possibility (but not in published $\mathrm{F}$ and $\mathrm{G}$ dwarf models).

In perfectly stable radiative layers, atomic diffusion of a given species must take place against the hydrogen background. Types of diffusion include gravitational settling (downward), thermal diffusion (downward), and radiative acceleration (upward; see, e.g., Michaud 1986 and 2000, these proceedings). Gravitational settling results from the fact that hydrostatic equilibrium varies slightly from species to species; thermal diffusion results from the dependence of atomic motion on atomic mass; and radiative acceleration is related to the different effective cross sections that result from different stages of ionization. However, even a small amount of turbulence can make the diffusion timescales extremely long and thus effectively decrease or even erase its signatures. Thus, no diffusion takes place within SCZ's. Furthermore, diffusion becomes increasingly efficient with decreasing density, i.e., increasing radius; thus, while little diffusion is predicted and indeed inferred from helioseismology in the Sun (Bahcall \& Pinsonneault 1996; Richard et al. 1996; Guenther et al. 1996; Vauclair 1998; Basu et al. 2000), more is predicted for hotter dwarfs. Diffusion clearly plays a role in creating peculiar abundances in some A stars and earlier types (Stephens \& Deliyannis 1999; Michaud et al. 1976, Michaud 1970, 2000, these proceedings, and references therein).

Mass loss has been invoked as a Li depletion mechanism in low mass PopI stars (Schramm et al. 1990; Guzik et al. 1987; Hobbs et al. 1989). The main idea is that the Li preservation region (Figure 1) is lost, so the surface abundance goes down (this is different from the suggestion by Vauclair \& Charbonnel 1995 for Pop II stars as discussed in Part II). In G stars the SCZ occupies a significant fraction of the Li preservation region, so a necessary complication to this scenario is the continuous dilution of $\mathrm{Li}$ as mass is lost. The required mass loss rates are orders of magnitude larger than what is observed in the Sun.

At least two types of slow mixing are possible. Gravity waves (Garcia Lopez \& Spruit 1991, Schatzman 1993, Montalban \& Schatzman 1994, Kumar \& Quar- 
tart 1997, Zahn et al. 1997, Kumar et al. 1999) can cause slow mixing below the SCZ when convective motions pound against the base of the SCZ. Slow mixing can also be rotationally-induced (Pinsonneault et al. 1990; Chaboyer \& Zahn 1992; Chaboyer et al. 1995; Vauclair et al. 1978, Vauclair 1988, Charbonnel et al. 1992, 1994, Charbonnel \& Vauclair 1992, Zahn 1992, Maeder 1995, Talon \& Zahn 1997, Talon \& Charbonnel 1998, Charbonnel \& Talon 1999, Brun et al. 1999) as stars losing angular momentum from their surfaces trigger instabilities in the interior that cause mixing. Two schools have been constructing detailed models that include rotational mixing of light elements, the Yale school and the French-Swiss school. Although some details differ, from a broad perspective, the two schools are much more similar than they are different (see Maeder \& Meynet 2000 for an indispensable and enlightening review).

Of course, it is also possible that other types of mechanisms, not discussed here, may cause depletion of light element abundances.

\section{The Cool Stars}

Like the Solar Neutrino problem, the Solar Li problem has been with us for over three decades. The problem is that the Solar $\mathrm{Li}$ abundance lies well below the Hyades Li morphology (Figure 2), yet classical models predict no main sequence Li depletion. Other old stars near the solar $\mathrm{T}_{\text {eff }}$ of $5780 \mathrm{~K}$, particularly 16 Cyg $\mathrm{A}$ and $\mathrm{B}$, and $\alpha$ Cen A show similarly low $\mathrm{Li}$ abundances (King et al. 1997, and references therein). The accumulating cluster evidence (Figure 2 , but also additional clusters discussed by Jeffries 2000) argues that G stars experience main sequence $\mathrm{Li}$ depletion: there is a good correlation between mean cluster $\mathrm{Li}$ and age. These data contradict one possible and often-visited solution, namely that of ad hoc convective overshoot (e.g. Straus et al. 1976). This effectively makes the SCZ deeper, allowing for more Li depletion. The problem is that convective overshoot preserves the basic time dependence of light element depletion in classical models, e.g. rapid pre-MS depletion and a sharp boundary between main sequence depletion and non-depletion; it does not permit main sequence depletion across a wide range of SCZ depths. Thus, if the solar Li is now matched, the predicted Hyades Li depletion (and that of most other clusters) is now too great. This issue also relates to the historical debate of the Hyades vs. Pleiades Li morphologies, dating back to when only these two clusters had good $\mathrm{G}$ dwarf Li data. Proponents of the classical model pointed out that the Hyades is metal-rich compared to the Pleiades, and should thus experience more pre-MS $\mathrm{Li}$ depletion. However, the preponderance of data in Figure 2 argues that the $\mathrm{Li}$ depletion is predominantly a main sequence phenomenon, in contrast to the classical theory. Specific examples can be cited. The Pleiades, M34, UMa, and M67 are all solar metallicity clusters and show an age-Li relation. NGC 752 is a factor of 2 more metal-poor than the Hyades, yet its Li Morphology lies below that of the Hyades. Regardless of the true explanation for $\mathrm{Li}$ depletion, this suggests that age is a more important $\mathrm{Li}$ depletion parameter than metallicity; it remains to be seen how much of a role (if any) metallicity plays in $\mathrm{G}$ dwarf $\mathrm{Li}$ depletion, at least near solar metallicity. At intermediate and halo metallicity, it is clear that $\mathrm{Li}$ depletion does depend on the metal content because then 
structural effects are sufficiently important that the mass scale has shifted to higher $\mathrm{T}_{\text {eff }}$.

Atomic diffusion at the $10 \%$ level is inferred from a comparison of solar models and helioseismic data, close to the magnitude expected from theoretical calculations. However, the atomic diffusion time scales are too long to explain the bulk of the $\mathrm{G}$ dwarf $\mathrm{Li}$ depletion, and the timing is inverted (diffusion rates should increase with age as the convective envelope gets thinner, but the rate of $\mathrm{Li}$ depletion depletion seems to decline with age). To explain the Hyades Li morphology using mass loss requires an unrealistic initial mass function (Swenson \& Faulkner 1992).

The most promising mechanism is slow mixing which is inferred from helioseismology (e.g., Christensen-Dalsgaard et al. 1993). Since there exist rotationage, activity-age, and Xray-age correlations, it is tempting to prefer rotational mixing over gravity wave mixing. Furthermore, higher Li abundances in short period binaries also points to the action of rotational mixing (Deliyannis et al. 1990, Deliyannis 1990, Zahn 1992, 1994, Cayrel de Strobel et al. 1994, Ryan \& Deliyannis 1995, Barrado y Navascues \& Stauffer 1996). However, both mechanisms require further theoretical investigation (see Talon \& Charbonnel 1998 for a discussion on the present description of the transport of angular momentum in low mass stars) and particularly, predictions should be made that might distinguish between the two types of slow mixing (see also $\S 6$ ).

\section{The Li Plateau}

The level of the $\mathrm{Li}$ abundance of the open cluster Li plateau appears to be lower with age, but the effect is not as dramatic as in the cooler $\mathrm{G}$ stars. In principle, this could reflect one of two things: Li depletion with age, or Galactic Li production (or both). Short period tidally locked binaries in the Hyades (Deliyannis et al. 1990; Soderblom et al. 1990; Thorburn et al. 1993; Ryan \& Deliyannis 1995) and in M67 (Deliyannis et al. 1994; Pasquini et al. 1997) have Li abundances that lie above the mean Li Morphology of those clusters. This argues that, at least in those clusters, the Li plateaus have been depleted, and supports rotationally-induced mixing as the cause of the Li depletion (see Deliyannis et al. 1990, Deliyannis 1990, Zahn 1992, 1994, Ryan \& Deliyannis 1995 for the detailed discussion of how such binaries can protect against rotational mixing).

The possible connection to the halo Li plateau is intriguing. Rotational models that reproduce the open cluster Li plateau depletion also suggest that the halo Li plateau is depleted. It is becoming increasingly clear that the depletion needs not be very large, but it also seems that if near-zero Li depletion has occured, halo dwarfs would have to be radically different than their disk counterparts. We refer to Part II for more discussion of this issue.

\section{The Li Gap}

The sharp decline of Li abundances in F stars offers, perhaps, the most striking evidence for the inadequacy of classical models. The Li Gap was first discovered in the Hyades (Boesgaard \& Tripicco 1986), and its absence in the Pleiades (Boesgaard et al. 1988) demonstrated that it is a main sequence phenomenon. 
At least three classes of mechanisms purport to explain it. Finely tuned (large) mass loss rates can, in principle, create the gap. Downward diffusion can create the red (cool) side of the gap; early models indicated that radiative acceleration might create the blue (hot) side of the gap, though improved later models did not show this (Michaud 1986; Richer \& Michaud 1993). Slow mixing, induced either by gravity waves or rotation, can also create the gap, by mixing the $\mathrm{Li}$ preservation region with material below that is devoid of $\mathrm{Li}$. Combinations of these mechanisms have also been proposed (e.g. Charbonneau \& Michaud 1988, Talon \& Charbonnel 1998). There are several ways to distinguish between these Li gap-producing mechanisms. Here, we focus on two methods: subgiants evolving out of the Li Gap, and information provided by additional elements, particularly $\mathrm{Be}$ and $\mathrm{B}$.

The subgiant method (see also Part III and Talon \& Charbonnel 1998 and in these proceedings) relies on determining the $\mathrm{Li}$ profile. Whereas all mechanisms predict a Li gap, the degeneracy is broken in the different signatures each mechanism leaves on the Li profile (Deliyannis et al. 1997). To deplete the surface $\mathrm{Li}$ abundance (in the presence of a very shallow SCZ), mass loss must get rid of essentially all of the $\mathrm{Li}$ preservation region (Figure 2). This leaves a tiny sliver of a Li preservation region, in which the Li abundance declines steeply with depth. For the red side of the gap, increasing diffusion rates with depth means $\mathrm{Li}$ piles up below the SCZ, and forms a peak. So Li increases with depth, but then decreases again at the Li preservation boundary. Rotationally-induced mixing generally flattens the Li profile (e.g. Deliyannis \& Pinsonneault 1997, Talon \& Charbonnel 1998), depending on the details of how the mixing occurs. These different profiles could be determined if a cluster existed with a turnoff in the Li gap: its subgiants would be evolving out of the gap, and as their convection zones deepen and dredge up progressively deeper material, they reveal the main sequence Li profile. Particularly, for mass loss, the subgiant surface $\mathrm{Li}$ abundances would decrease very sharply with decreasing $\mathrm{T}_{e f f}$. For diffusion, the $\mathrm{Li}$ abundances would increase at first, and then decrease at cooler $\mathrm{T}_{\text {eff }}$. The case of slow mixing would lie in between the other two cases.

Fortuitously, nature provides a nearby rich cluster with turnoff on the cool side of the Li gap, namely M67. WIYN/Hydra observations of numerous stars near the turnoff at resolution $\mathrm{R} \sim 15,000=3$ pixels and $\mathrm{S} / \mathrm{N}$ (of order 200-300 per pixel) are able to address this issue; models were also specifically constructed using the Yale/Ohio State code to interpret the observations (Deliyannis et al. 1997; Sills \& Deliyannis 2000). These models were calibrated to create the Hyades Li gap, and took into account the metallicity difference between the two clusters. The subgiant abundances drop quickly from their turnoff value of $\mathrm{A}(\mathrm{Li})=2.4$, and also exhibit a significant spread. Mass loss is generally argued against in that straightforward scenarios that explain one cluster fail to explain the other; however, some scenarios with time-dependent mass loss rates might, conceivably, still be viable. Even these, though, are ruled out by the $\mathrm{Li}-\mathrm{Be}$ depletion correlation (below). Pure diffusion is clearly argued against, except possibly for the intriguing case of one star. Rotational mixing might possibly be consistent with the observations if the observations are exhibiting large $\mathrm{Li}$ spreads, rather than a steeply declining $\mathrm{Li}$ abundance. In the case of the latter, however, the models do not have a sufficiently steep Li profile. We refer to Part III for further discussion concerning field subgiants. 

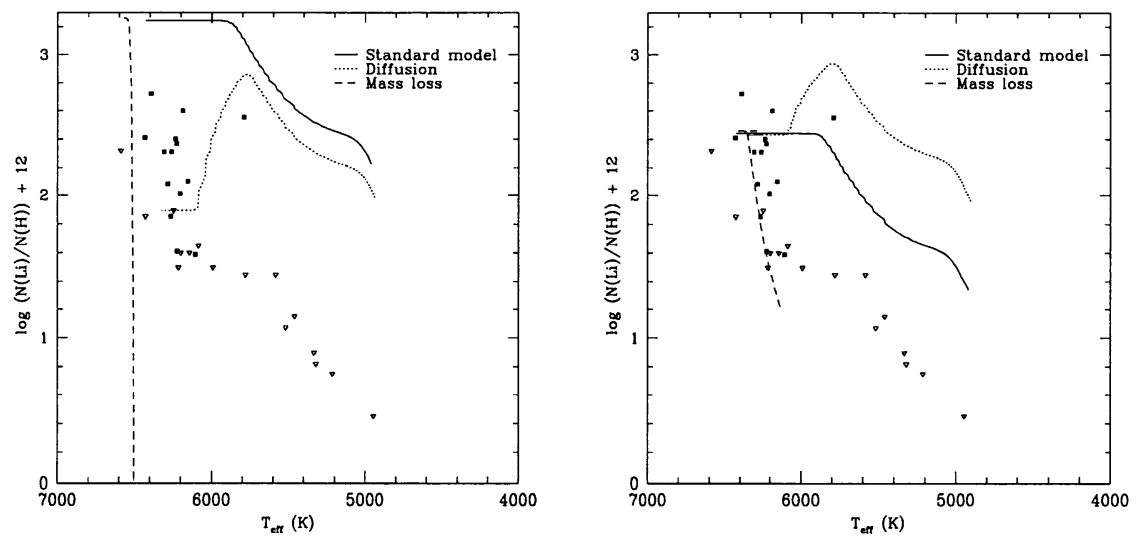

Figure 3. WIYN/Hydra MOS Li observations in M67 subgiants near the turnoff, compared to various models. Left: the models are calibrated to the Hyades Li gap depletion. Right: the models are calibrated to the M67 abundance just below the turnoff.

Very powerful tests of the models are realized when one uses more than one light element simultaneously. Consider using $\mathrm{Li}$ and $\mathrm{Be}$. Whereas all classes of mechanisms predict a $\mathrm{Li} \mathrm{Gap}$, the Be signatures vary from mechanism to mechanism (Deliyannis \& Pinsonneault 1997; Deliyannis et al. 1998a, Talon \& Charbonnel 1998). In the case of mass loss, there is no change in $\mathrm{Be}$ as the surface Li goes down (Figure 1). All detectable Li must long be gone before any Be depletion occurs. In the case of pure diffusion, all the action takes place at the base of the SCZ: both elements diffuse downwards at similar rates. In the case of slow mixing, both the $\mathrm{Li}$ and Be profiles are flattened, and the surface abundances of both elements decline simultaneously. Li goes down faster than $\mathrm{Be}$, depending on how the mixing occurs. In one extreme case, Li goes down as little as twice as fast as $\mathrm{Be}$ - the ratio of the sizes of their preservation regions. In models with rotational mixing, the efficiency of mixing increases with radius, and as a result Li goes down a little more than 4 times faster than Be does; both the Yale and French models agree on this (Deliyannis \& Pinsonneault 1997; Charbonnel et al. 1994, Talon \& Charbonnel 1998). In models with gravity waves (Garcia Lopez \& Spruit 1991) the mixing is confined to smaller regions near the SCZ, so Li goes down at an even faster rate relative to Be. Thus, in a plot of $\mathrm{A}(\mathrm{Be})$ vs. $\mathrm{A}(\mathrm{Li})$, mass loss would be a horizontal line, diffusion would be a 45-degree line, rotational slow mixing would be shallower, and wave-driven slow mixing would be even shallower.

Figure 4 shows data (left panel) of a $\mathrm{Li}$ and Be survey of field $\mathrm{F}$ stars aimed specifically at identifying which mechanism creates the Li Gap (left panel; Deliyannis et al. 1998a). Great care was taken to define empirically the undepleted $\mathrm{Li}$ and $\mathrm{Be}$ abundances, by observing $\mathrm{Be}$ in stars that have $\mathrm{A}(\mathrm{Li})$ greater than 3.0 (rather than assume, as is often done, that stars with lower Li will have undepleted $\mathrm{Be}$ ), and to get detections in $\mathrm{Li}$ at low $\mathrm{A}(\mathrm{Li}) \sim 1$. The data show a correlation between the depletion of $\mathrm{Li}$ and $\mathrm{Be}$. This is remarkable in view of the 


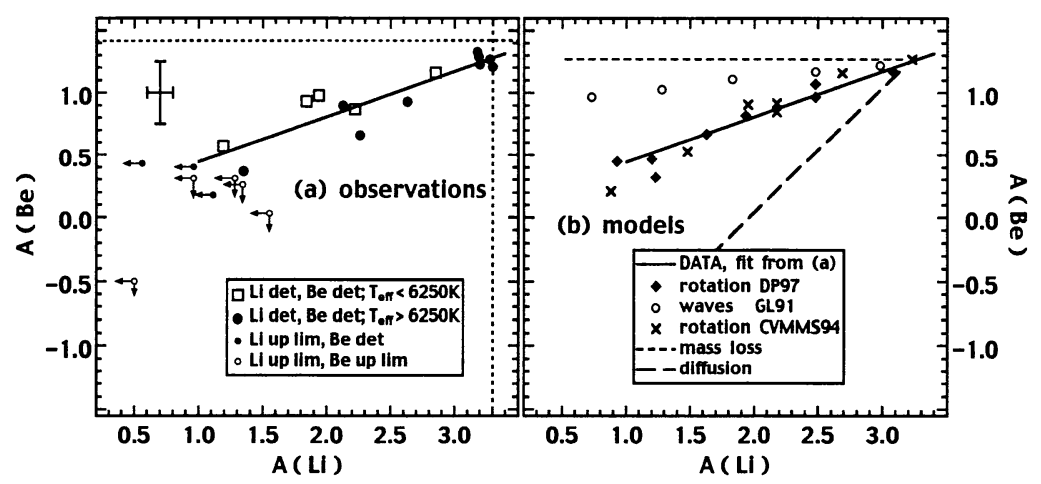

Figure 4. Keck I/HIRES, CFHT/Gecko, and UH 2.2m/Coudé observations of $\mathrm{Li}$ and $\mathrm{Be}$ in field $\mathrm{F}$ and $\mathrm{G}$ stars, illustrating a correlated depletion of surface $\mathrm{Li}$ and $\mathrm{Be}$. This argues strongly against mass loss and diffusion, and in favor of slow mixing as the dominant physical mechanism that creates the Li gap. Rotationally-induced mixing is favored over mixing induced by gravity waves.

fact that many of the stars in the sample are the same stars that show chaotic $\mathrm{Li}$ abundances in Figure 1, with a variety of different ages. Figure $4 \mathrm{~b}$ shows that this $\mathrm{Li}-\mathrm{Be}$ depletion correlation argues strongly against mass loss and pure diffusion. Rotational mixing is strongly supported. Wave driven mixing does not do too badly, but the data favor rotational mixing. Note that the slope is slightly shallower for cooler stars, as predicted by the rotational models: this is due to the fact that cooler stars have deeper SCZ's which thus do a greater share of the mixing, leaving rotational instabilities free to deplete a larger amount of $\mathrm{Li}$ for a given $\mathrm{Be}$ depletion (since $\mathrm{Be}$ is preserved to a deeper level, convection does a smaller fraction of Be mixing than $\mathrm{Li}$ mixing). It is worth emphasizing the power of this method: not only does it identify the general class of model responsible for the $\mathrm{Li}$ gap, but it also seems able to distinguish between two types of slow mixing. It should be noted that we have identified the dominant mechanism; it is still possible that other mechanisms also occur to a more modest degree. In view of these findings, it is understandable why pure diffusion is not favored as the dominant mechanism, if mixing is favored: the mixing probably renders diffusion much less efficient. Future studies utilizing both $\mathrm{Li}$ and Be should be able to provide further constraints on slow mixing, especially if these studies include cluster stars; there is hope that the dominant mechanism can be understood in detail. Finally, a limited number of B observations show an essentially constant $\mathrm{B}$ abundance for highly varying degrees of $\mathrm{Li}$ and Be depletion (Boesgaard et al. 
1998); this provides yet another independent argument against diffusion acting alone, and restricts the depth to which material can be mixed ${ }^{1}$.

\section{Conclusions}

Classical stellar evolution theory fails to explain nearly all patterns of $\mathrm{Li}, \mathrm{Be}$, and B abundances in Population I low mass stars. Although several physical mechanisms have been proposed, the preponderance of data favor slow mixing, probably induced by rotation, that must compete with atomic diffusion. Future work should improve understanding of this slow mixing and evaluate what secondary role (if any) is played by wave-induced mixing, mass loss, and magnetic fields.

\section{Acknowledgements}

C.P.D. acknowledges support from the United States National Science Foundation under grant AST-9812735. M.H.P. acknowledges support from the United States National Science Foundation under grant AST-9731621 and from NASA under grant NAG5-7150. C.C. acknowledges support from the Action Spécifique de Physique Stellaire and the Conseil National Français d'Astronomie.

\section{References}

Barrado y Navascues, D. \& Stauffer, J. R. 1996, A\&A, 310, 879

Basu, S., Bahcall, J.N., \& Pinsonneault, M.H. 2000, ApJ, 529, 1084

Boesgaard, A. M., \& Tripicco, M. J. 1986a, ApJ, 302, L49

Boesgaard, A. M., \& Tripicco, M. J. 1986b, ApJ, 303, 724

Boesgaard, A. M., Budge, K. G., \& Ramsay, M. E. 1988, ApJ, 327, 389

Boesgaard, A. M., Deliyannis, C. P., Stephens, A., \& Lambert, D. L. 1998, ApJ, $492,727$.

Brun, A.S., Turck-Chièze, S., Zahn, J.P., 1999, ApJ525, 1032

Cayrel, R. 1999, in "LiBeB, Cosmic Rays, and Related X- and Gamma-Rays,", eds. R. Ramaty, E. Vangioni-Flam, M. Casse, and K. Olive, ASPCS 171, p. 268

Cayrel de Strobel, G., Cayrel, R., Friel, E., Zahn, J.P., Bentolila, C., 1994, A\&A, 291,505

Chaboyer, B., Zahn, J.P., 1992, A\&A253, 173

Chaboyer, B., Demarque, P., \& Pinsonneault, M. H. 1995, ApJ, 441, 876.

Charbonnel, C. \& Talon, S., 1999, A\&A, 351, 635

Charbonnel, C. \& Vauclair, S. 1992, A\&A, 265, 55

\footnotetext{
${ }^{1}$ In addition, the carbon and ozygen underabundances which are expected in the case of pure diffusion was not found in the Hyades F stars (Friel \& Boesgaard 1990, Garcia Lopez et al. 1993)
} 
Charbonnel, C., Vauclair, S. \& Zahn, J.-P. 1992, A\&A, 255, 191

Charbonnel, C., Vauclair, S., Maeder, A., Meynet, G., \& Shaller, G. 1994, A\&A, 283,155

Christensen-Dalsgaard, J., Proffitt, C.R., Thompson, M.J., 1993, ApJ403, L75

Charbonneau, P., \& Michaud, G. 1988, ApJ, 334, 746

D'Antona, F. \& Mazzitelli, I. 1994 ApJS, 90, 467

Deliyannis, C. P., Ph.D. Dissertation, Yale University

Deliyannis, C. P., Demarque, P., \& Kawaler, S. 1990, ApJS, 73, 21

Deliyannis, C. P., King, J., Boesgaard, A.M, \& Ryan, S.G. 1994, ApJ, 434, L71

Deliyannis, C. P., \& Pinsonneault, M. H. 1997, ApJ, 488, 836

Deliyannis, C. P., King, J., \& Boesgaard, A. M. 1997, in "Wide Field Spectroscopy", eds. E. Kontizas et al., (Kluwer), Ast. \& SSL 212, p. 201

Deliyannis, C. P., Boesgaard, A. M., Stephens, A., King, J. R., Vogt, S. S., \& Keane, M. J. 1998a, ApJ, 498, L147

Deliyannis, C. P., Boesgaard, A. M., Stephens, A., King, J. R., Vogt, S. S., \& Keane, M. J. 1998b, in "Nuclei in the Cosmos V", eds. N. Prantzos \& S. Harissopoulos, Editions Frontieres, p. 41

Deliyannis, C. P. 2000, in "Stellar Clusters and Associations: Convection, Rotation, and Dynamos", eds. R. Pallavicini, G. Micela, \& S. Sciortino, ASPCS 198, p. 235

Forestini, M 1994, A\&A, 285, 473

Friel, E.D., Boesgaard, A.M., 1990, ApJ351, 480

Garcia López, R. J., Rebolo, R., Herrero, A., Beckman, J.E., 1993, ApJ412, 173

Garcia López, R. J., \& Spruit, H. C. 1991, ApJ, 377, 268

Guenther, D. B., Kim, Y.-C., \& Demarque, P., 1996, ApJ, 463, 382

Guzik, J.A., Willson, L.A., Brunish, W.M., 1987, ApJ, 319, 957

Hobbs, L. M., Iben, I., \& Pilachowski, C. 1989, ApJ, 347, 817

Jeffries, R. D. 2000, in "Stellar Clusters and Associations: Convection, Rotation, and Dynamos", eds. R. Pallavicini, G. Micela, \& S. Sciortino, ASPCS 198 , p. 245

Jones, B. F., Fischer, D., Shetrone, M., Soderblom, D. R., 1997, AJ, 114, 352

King, J. R., Deliyannis, C. P., Hiltgen, D. D., Stephens, A., Cunha, K., \& Boesgaard, A. M. 1997, AJ, 113, 1871

Kumar, P., Quartaert, E.J., 1997, ApJ, 475, L143

Kumar, P., Talon, S., Zahn, J.P., 1999, ApJ, 520, 859

Maeder, A., 1995, A\&A, 299, 84

Maeder, A., Meynet, G., 2000, ARAA

Martin, E. L. 1997, Mem.S.Ast.It. 68, p. 905

Mathieu, R. 2000, in "Stellar Clusters and Associations: Convection, Rotation, and Dynamos", eds. R. Pallavicini, G. Micela, \& S. Sciortino, ASPCS 198, p. 517

Michaud G., 1970, ApJ, 160, 641

Michaud, G. 1986, ApJ, 302, 650 
Michaud, G., Charland, Y., Vauclair, S., Vauclair, G., 1976, ApJ, 210, 447

Montalban, J., Schatzman, E., 1994, A\&A, 305, 513

Pasquini, L., Randich, S., \& Pallavicini, R. 1997, A\&A, 325, 535

Pilachowski, C. A., Booth, J., \& Hobbs, L. M. 1987, PASP, 99, 1288

Pinsonneault, M. H. 1997, ARA\&A, 35, 557

Pinsonneault, M. H., Kawaler, S. D., \& Demarque, P. 1990, ApJS, 74, 501

Proffitt, C. R., \& Michaud, G. 1989, ApJ, 346, 976

Richard, O., Vauclair, S., Charbonnel, C., Dziembowski, W.A. 1996, A\&A, 312, 1000

Richer, J., \& Michaud, G. 1993, ApJ, 416, 312

Ryan, S. G., \& Deliyannis, C. P. 1995, ApJ, 453, 819

Schatzman, E., 1993, A\&A279, 431

Schramm, D. N., Steigman, G., \& Dearborn, D. S. P. 1990, ApJ, 259, L55

Sills, A., \& Deliyannis, C. P. 2000, ApJ, submitted.

Soderblom, D., Oey, M., Johnson, D., \& Stone, R., 1990, AJ, 99, 595

Soderblom, D., Jones, B., Balachandran, S., Stauffer, J., Duncan, D., Fedele, S., \& Hudon, J. 1993a, AJ, 106, 1059.

Soderblom, D., Fedele, S., Jones, B., Stauffer, J., \& Prosser, C. 1993b, AJ, 106, 1080.

Stephens, A., \& Deliyannis, C. P. 1999, PASP, 758, 482

Straus, J. M., Blake, J. B., \& Schramm, D. N. 1976, ApJ, 204, 481

Swenson, F.J. \& Faulkner, J. 1992, ApJ, 395, 654

Talon, S., Charbonnel, C., 1998, A\&A, 335, 959

Talon, S., Zahn, J.P. 1997, A\&A, 317, 749

Thorburn, J. A., Hobbs, L. M., Deliyannis, C. P., \& Pinsonneault, M. H. 1993, ApJ, 415, 150

Vauclair, G., Vauclair, S., Michaud, G., 1978, ApJ223, 920

Vauclair, S. 1988, ApJ335, 971

Vauclair, S. 1998, Space Sci.Rev., 85, 71

Zahn, J.-P., 1992, A\&A, 265, 115

Zahn, J.-P., 1994, A\&A, 288, 829

Zahn, J.-P., Talon, S., Matias, J., 1997, A\&A, 332, 320 